\title{
ANGLE-RESOLVED PHOTOEMISSION EXTENDED FINE STRUCTURE: MULTIPLE LAYERS OF EMITTERS AND MULTIPLE INITIAL STATES*
}

W.R.A Huffa,b, Y. Chenc, S.A. Kellara,b, E.J. Molera, b, H. Wuc, Z. Hussaina, D.A. Shirleyc

${ }^{a}$ Advanced Light Source

Lawrence Berkeley National Laboratory

University of California

Berkeley, CA 94720

bThe University of California

Department of Chemistry

Berkeley, CA 94720

cThe Pennsylvania State University

Department of Chemistry and Physics

University Park, PA 16802

Paper submitted at the 11th International Conference on Vacuum Ultraviolet Radiation Physics, Tokyo, Japan, August 27 - September 1, 1995

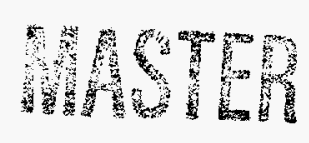

*This work was supported by the Director, Office of Energy Research, Office of Basic Energy Sciences, Materials Sciences Division, of the U.S. Department of Energy, under Contract No. DE-AC03-76SF00098. 
(2) 


\section{DISCLAIMER}

This report was prepared as an account of work sponsored by an agency of the United States Government. Neither the United States Government nor any agency thereof, nor any of their employees, make any warranty, express or implied, or assumes any legal liability or responsibility for the accuracy, completeness, or usefulness of any information, apparatus, product, or process disclosed, or represents that its use would not infringe privately owned rights. Reference herein to any specific commercial product, process, or service by trade name, trademark, manufacturer, or otherwise does not necessarily constitute or imply its endorsement, recommendation, or favoring by the United States Government or any agency thereof. The views and opinions of authors expressed herein do not necessarily state or reflect those of the United States Government or any agency thereof. 


\section{DISCLAIMER}

\section{Portions of this document may be illegible in electronic image products. Images are produced from the best available original document.}



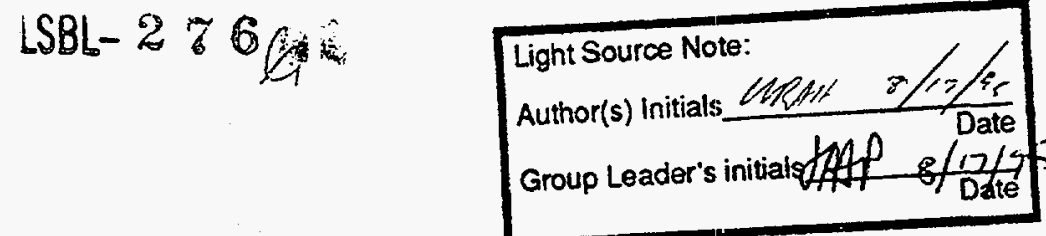

\title{
Angle-Resolved Photoemission Extended Fine Structure: Multiple Layers of Emitters and Multiple Initial States
}

\author{
W.R.A. Huff ${ }^{a, b}$, Y. Chen ${ }^{c}$, S.A. Kellar ${ }^{a, b}$, E.J. Moler ${ }^{a, b}$, H. Wu $u^{c}$, Z. Hussain ${ }^{b}$, and D.A. Shirley \\ ${ }^{a}$ Lawrence Berkeley National Laboratory, Berkeley, CA 94720 \\ ${ }^{b}$ The University of California, Dept. of Chemistry, Berkeley, CA 94720 \\ 'The Pennsylvania State University, Dept. of Chemistry and Physics, University Park, PA 16802
}

Recently, angle-resolved photoemission extended fine structure (ARPEFS) has been applied to experimental systems involving multiple layers of emitters and non-s core-level photoemission in an effort to broaden the utility of the technique. Most of the previous systems have been comprised of atomic or molecular overlayers adsorbed onto a single-crystal, metal surface and the photoemission data were taken from an $s$ atomic core-level in the overlayer. For such a system, the acquired ARPEFS data is dominated by the $p_{0}$ final state wave backscattering from the substrate atoms and is well understood. In this study, we investigate ARPEFS as a surface-region structure determination technique when applied to experimental systems comprised of multiple layers of photoemitters and arbitrary initial state core-level photoemission. Understanding the data acquired from multiple layers of photoemitters is useful for studying multiláyer interfaces, "buried" surfaces, and clean crystals in ultra-high vacuum. The ability to apply ARPEFS to arbitrary initial state core-level photoemission obviously opens up many systems to analysis. Efforts have been ongoing to understand such data in depth. We present clean $\mathrm{Cu}(111)$ $3 s, 3 p$, and $3 d$ core-level, normal photoemission data taken on a high resolution soft $\mathrm{x}$-ray beamline 9.3 .2 at the Advanced Light Source in Berkeley, California and clean Ni(111) $3 p$ normal photoemission data taken at the National Synchrotron Light Source in Upton, New York, USA.

\section{INTRODUCTION}

Angle-resolved photoemission extended fine-structure (ARPEFS) is a proved technique for determining surface structures of metal and nonmetal atomic adsorbate systems as well as molecular adsorbates on conducting single crystal surfaces. ARPEFS yields accurate information about both the local structure around the adsorbates and the adsorbate-induced relaxation of the substrates. Most of the previous ARPEFS studies were based on photoemission data from atomic $s$ core-level initial states, for which the selection rules $\Delta \ell_{\mathrm{i}}= \pm 1$, and $\Delta m_{\mathrm{i}}=0$ give a $p_{0}$-wave final state. Our experience with ARPEFS data from non-s initial states and their Fourier transforms (FTs) is very limited, however.! For non-s initial states $\left(\ell_{i} \neq 0\right)$, the photoelectron final state is made up of partial waves with orbital quantum numbers $\ell_{i}+1$ and $\ell_{i}-1$, and a phase relationship between them which leads to interference between the partial waves. Note that the allowed $m$ levels will be populated in the final state. Thus, with a $p$ initial state, the partial waves consist of $\ell_{\mathrm{f}}=0, m=0$ as well as $\ell_{\mathrm{f}}=2$, $m=0, \pm 1$. With a $d$ initial state, the partial waves consist of $\ell_{\mathrm{f}}=1, m=0, \pm 1$ as well as $\ell_{\mathrm{f}}=3$, $m=0, \pm 1, \pm 2$. The partial wave radial dipole matrix elements and the phase shifts are in general energydependent. Despite these complications, there are a number of interesting experimental situations for which ARPEFS studies on a non-s initial state may confer some advantage.

Our purpose here is to explore the applicability of ARPEFS to non- $s$ initial state photoemission of clean surfaces with the ultimate goal of developing a method for studying photoemission from an arbitrary initial state as well as determine the atomic structure of interfaces, for which ARPEFS seems ideally suited. In favorable cases, atomic relaxation and reconstruction could be studied as well. In such studies, the elemental and chemical specificity of ARPEFS and its sensitivity to atomic layers that are several layers below the surface offer certain benefits.

\section{EXPERIMENTAL}

The $\mathrm{Cu}$ experiments were performed at the ALS BL 9.3.2 in an ultra-high vacuum chamber (pressure $\sim 6 \mathrm{nPa}$ ) equipped with standard UHV surface science sample cleaning and preparation tools. The $\mathrm{Cu}$ crystal was mounted on an $x, y, z, \theta, \phi$ manipulator equipped with a liquid helium cooled cryostat operating at $100 \mathrm{~K}$. The Ni experiment was performed at the NSLS on BLU3-C where the 
manipulator was cooled with $\mathrm{LN}_{2}$ to $100 \mathrm{~K}$. The crystals were cleaned by repetitive cycles of argon ion sputtering and subsequent annealing by electron bombardment from behind to $700{ }^{\circ} \mathrm{C}$. Sample cleanliness was monitored using $\mathrm{x}$-ray photoelectron spectroscopy (XPS) and checking for $\mathrm{C} 1 s, \mathrm{O} 1 s$, and $\mathrm{S} 2 p$; no contamination was detected before or after the data collection which lasted five hours for each $\mathrm{Cu}$ data set and nine hours for the Ni data set.

The XPS spectra were collected using an angle-resolving (acceptance angle $\pm 2^{\circ}$ ) electrostatic hemispherical electron energy analyzer oriented normal to the crystals' (111) surface. The photon polarization vector was oriented $10^{\circ}$ (for $\mathrm{Cu}$ data) and $35^{\circ}$ (for $\mathrm{Ni}$ data) from the surface normal.

\section{DATA REDUCTION}

ARPEFS raw data are a series of XPS spectra with changing photoelectron kinetic energy from $-95 \mathrm{eV}$ to $-550 \mathrm{eV}$. Using the de Broglie relation $k\left(\AA^{-1}\right)=0.5123 \sqrt{E(\mathrm{eV})}$, this photoelectron energy range corresponds to the magnitude of the photoelectron wave vector range $5 \AA^{-1}$ to $12 \AA^{-1}$. From this, a useful wave vector range will be determined by the specific experimental conditions. The spectra are typically recorded in equal steps of $0.1 \AA^{-1}$. Note that this is the wave vector as measured by the analyzer (outside of the crystal); the scattering takes place inside the crystal and the ARPEFS curve must be adjusted for the inner potential of the solid before taking the FT.

Each XPS spectrum was a window encompassing the respective core-level photoemission peak(s). The peak(s) were fit with a voigt function to model the natural linewidth and the experimental broadening respectively. This voigt function was added to a Fermi step function whose intensity was scaled to the respective peak intensity to model the Shirley background of each peak. The width of the step was taken as the Gaussian width of the respective peak.

The purpose of fitting the spectra is to extract the most accurate area from the peaks to construct the $\chi(k)$ diffraction curve containing the structural information. $\chi(k)$ is defined by ${ }^{2}$

$\chi(k)=\frac{I(k)}{I_{0}(k)}-1$ where $I(k)$ is the peak area plotted as a function of the peak position in $k$-space. $I_{0}(k)$ is a smooth, slowly varying function with an oscillation frequency much lower than $I(k)$ and stems from the contribution of the inelastic scattering processes and the varying atomic cross section. Removing $I_{0}(k)$ results in a removal from the $\chi(k)$ FT the peaks $\leq 2 \AA$. Note that this study is of clean surfaces and thus photoemission occurred from surface atoms as well as atoms several layers below the surface. Many forward scattering path-length differences (PLDs) from sub-surface emitting atoms will be on the order of $\leq 2 \AA$ also. These forward scattering effects are therefore removed during the data reduction along with the standard $I_{0}(k)$; ARPEFS is thus a backscattering phenomenon.

\section{CAlculations}

Modeling calculations were performed in an attempt to simulate the ARPEFS $\chi(k)$ curve and obtain a structure more precise than yielded by the FT analysis. Using the single-scattering model of ARPEFS $^{2}, \chi(k)$ can be written as

$$
\chi(k)=\sum_{j} A_{j}(k) \cos \left[k\left(R_{j}-R_{j} \cos \theta_{j}\right)+\phi_{j}\right]
$$

where $A_{j}(k)$ contains experimental geometry factors including the photon polarization direction and the electron emission direction as well as the scattering amplitude, aperture integration, and thermal averaging. $\phi_{j}$ is the scattering phase shift.

A new code developed by $\mathrm{Wu}, \mathrm{Chen}$, and Shirley ${ }^{3}$ based on the Rehr-Albers formalism ${ }^{4}$ was used for the calculations presented here. This new code differs from the Kaduwela/Fadley ${ }^{5}$ code and is sufficiently fast that fitting calculations can be performed for systems in which the photoemitters are in many layers and the core-level initial state has arbitrary angular momentum.

For the calculations presented here, the radial dipole matrix elements and phase shifts were calculated in situ by using the atomic potentials tabulated in Ref. 6. These values describe the shape and phase relationship between the two partial waves, $\ell_{i} \pm 1$, and thus the true $s+d$ (or $p+f$ ) final 
state as a function of the photoemitted electron kinetic energy.

The multiple-scattering spherical-wave calculations require both nonstructural and structural input parameters. Besides the initial state and the matrix element tables, the nonstructural parameters included were the crystal temperature, the atomic scattering phase shifts, the inelastic mean free path, the emission and polarization directions, the electron analyzer acceptance angle, and the inner potential. The initial structural parameters used were those for a bulk-terminated fcc crystal. The fitting procedure allowed the structure to vary as well as the crystal temperature, the Debye temperature, and the inner potential such that a best fit was obtained and the applicability of ARPEFS could be assessed. The atomic-scattering phase shifts were calculated in situ by using the atomic potentials tabulated in Ref. 6 . The emission and polarization directions and the electron analyzer acceptance angle were set to match the experiment as described earlier. The inelastic mean free path (IMFP) was included using the exponential damping factor $e^{-r / \lambda}$ where $\lambda$ was calculated using the TPP- 2 formula derived in Ref. 7. It is expected that the IMFP calculation is important in obtaining a close fit to the data. The TPP-2 formula seems to be the most accurate method to determine the MMF, especially below

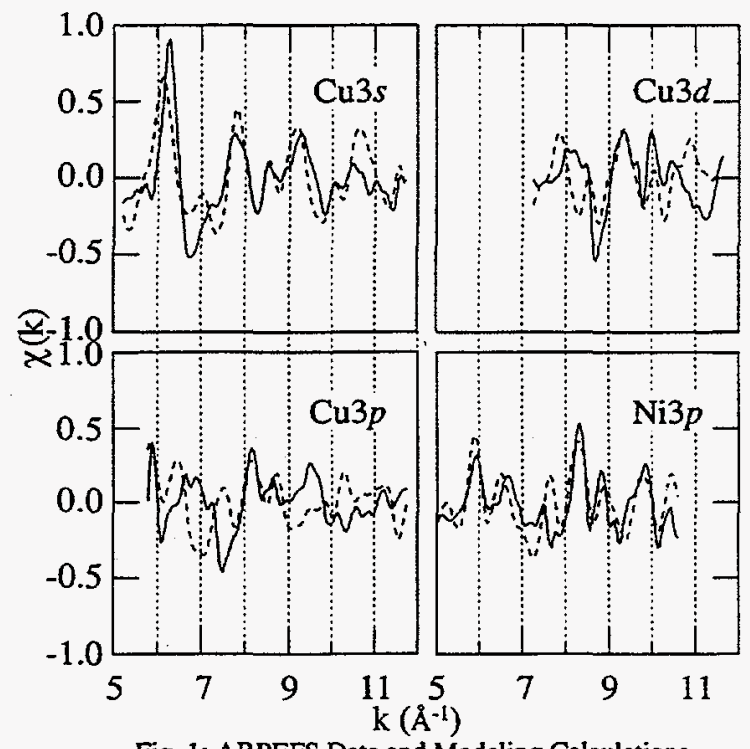

Fig. 1: ARPEFS Data and Modeling Calculations (solid Line is data, dashed line is fit)
$200 \mathrm{eV}$. Certainly, many emitters lie so deeply below the surface region that their signal never escapes the crystal.

\section{DISCUSSION}

The ARPEFS data and modeling calculations are plotted in Fig. 1 (solid line is data, dashed line is fit). These best fits were obtained with $T_{\text {Debye }}=350 \mathrm{~K}$ and inner potential $=10.5 \mathrm{eV}$. The fit to the $\mathrm{Cu} 3 s$ data is good, the $\mathrm{Cu} 3 d$ data is approximated, but the $\mathrm{Cu} 3 p$ data is not at all in agreement with the calculations. However, the $\mathrm{Ni} 3 p$ data agrees well with the modeling calculation. It is not yet clear why the $\mathrm{Ni} 3 p$ data can be well fit but the $\mathrm{Cu} 3 p$ data cannot. The similarities between the systems, regarding the scattering factors, phase shifts, lattice constants, etc., are such that the ARPEFS data and modeling are expected to yield very similar results. Experiments are planned to investigate this further.

There are two indications that the data are reasonable. One is that the $\mathrm{Cu} 3 s$ data and the $\mathrm{Cu} 3 p$ data are distinctly $180^{\circ}$ out of phase. ${ }^{1}$ Following the solid data curves, note that when one is at a maximum the other is at a minimum and visa versa. The FT analysis also indicates that the data. are reasonable (see Fig. 2).

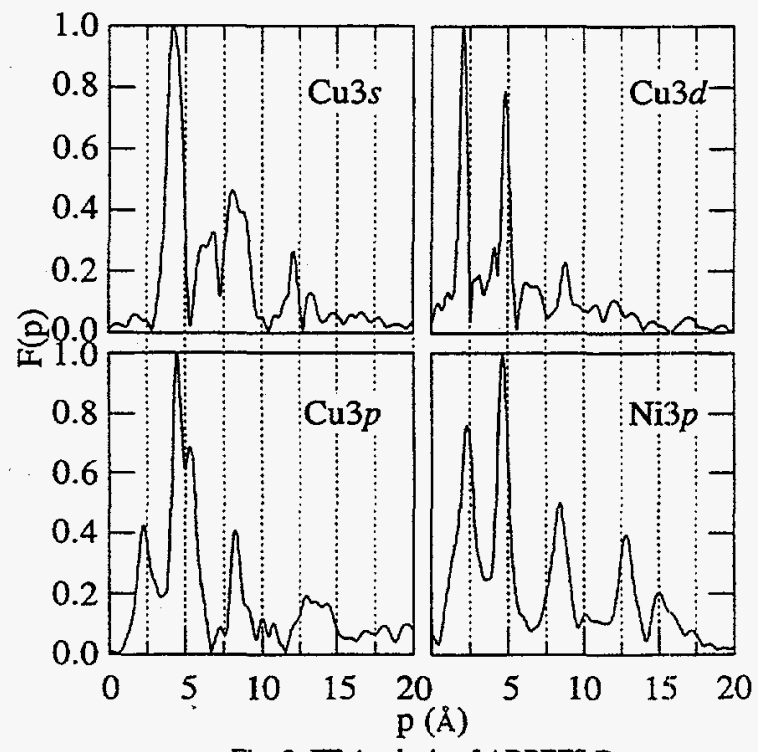

Fig. 2: FT Analysis of ARPEFS Data 
LEED studies have indicated a $0.03 \AA$ contraction of the $\mathrm{Cu}$ surface layer. ${ }^{8}$ The best fits plotted here average to a $0.08 \AA$ contraction.

However, before the ARPEFS results can be trusted, at least one off-normal ARPEFS study must be completed to compliment the normal emission data and the fitting must be improved.

The FTs plotted in Fig. 2 are similar for each data set and the peaks occur at reasonable pathlength differences between the direct and the scattered photoelectron waves based on the fcc crystal structure of the $\mathrm{Cu}(111)$ and $\mathrm{Ni}(111)$ samples. The peak at $-2.3 \AA$ in the $3 p$ FTs is indicative of scattering from the six nearest neighbors in the same layer as the emitter. This result has not been seen for $s$ initial state ARPEFS data and is believed to be forbidden by the symmetry of the $p_{0}$ final state wave function. This result should also be forbidden by symmetry for the $d$ initial state ARPEFS data; the peak at $2.1 \AA$ in the $\mathrm{Cu} 3 d \mathrm{FT}$ is due to forward scattering. It is shifted too far from the $2.55 \AA$ expected position based on the $\mathrm{Cu}$ lattice constant $(3.61 \AA)$ to be analogous to the peak in the $\mathrm{Cu} 3 p$ FT.

\section{CONCLUSION}

We report an ARPEFS study of the clean $\mathrm{Cu}(111)$ and $\mathrm{Ni}(111)$ surfaces where normal photoemission data were taken from the $\mathrm{Cu} 3 s, \mathrm{Cu} 3 p$, $\mathrm{Cu} 3 d$, and $\mathrm{Ni} 3 p$ atomic core-levels with the ultimate goal of developing a method for studying photoemission from an arbitrary initial state as well as determine the atomic structure of interfaces. The ARPEFS data resemble data for adsorbate systems and show strong backscattering signals from atoms up to four layers below the source atoms. Interface and multilayer systems are thus well suited for study by ARPEFS.

The ARPEFS data from this clean surface study and non- $s$ core-level initial state(s) agree with previous ARPEFS studies such that the backscattering cone model is supported by this work. The ARPEFS intensity can be regarded as arising from the sum of contributions from source atoms in each layer as if it were the surface layer. If we neglect forward scattering from atoms in layers above the source atoms, the ARPEFS intensity is modulated due to backscattering from the atoms in layers below the source atoms. Due to the finite mean free path, the signal from the sub-surface layer atoms is damped. It is important to note that photoelectron holography signals from clean surfaces are dominated by forward scattering, with atomic positions being imaged up to three layers ahead of the source atom. ${ }^{9}$ A combination of these two photoelectron diffraction techniques would therefore provide a very good method for studying ordered interfaces.

\section{ACKNOWLEDGMENTS}

The experiments were performed at the Advanced Light Source at Lawrence Berkeley National Laboratory and the National Synchrotron Light Source at Brookhaven National Laboratory. Both labs are supported by the U. S. Department of Energy's Office of Basic Energy Sciences. This work was supported by the DOE Contract No. DEAC03-76SF00098.

\section{REFERENCES}

${ }^{1}$ W.R.A. Huff, Y. Zheng, Z. Hussain, and D.A. Shirley, J. Phys. Chem. 98, 9182 (1994), and references therein.

2J.J. Barton, S.W. Robey, and D.A. Shirley, Phys. Rev. B 34, 778 (and 3807) (1986).

${ }^{3} \mathrm{H}$. Wu, Y. Chen, and D.A. Shirley, Unpublished.

${ }^{4}$ J.J. Rehr and R.C. Albers, Phys. Rev. B 41, 8139 (1990).

${ }^{5} \mathrm{~A}$. Kaduwela $\mathrm{PhD}$ Thesis, University of Hawaii at Manoa, Honolulu, 1991.

${ }^{6}$ V.L. Moruzzi, J.F. Janak, and A.R. Williams, "Calculated Electronic Properties of Metals." Pergamon Press, Inc., New York (1978).

${ }^{7}$ S. Tanuma, C. J. Powell, and D. R. Penn, Surf. Interface Anal. 20, 77 (1993).

${ }^{8}$ S.A. Lindgren, L. Wallden, J. Rundgren, and P. Westrin, Phys. Rev. B 29, 576 (1984).

${ }^{9}$ B. L. Petersen, J. L. Terminello, J. J. Barton, and D. A. Shirley, Chem. Phys. Lett. 213, 412 (1993). 